\title{
Combined effects of zoledronate and mechanical stimulation on bone adaptation in an axially loaded mouse tibia
}

\author{
V.A. Stadelmann ${ }^{\mathrm{a}, \mathrm{c}}$, N. Bonnet ${ }^{\mathrm{b}}$, D.P. Pioletti ${ }^{\mathrm{a}, *}$ \\ a Laboratory of Biomechanical Orthopedics, Ecole Polytechnique Fédérale de Lausanne, Switzerland \\ b Service of Bone Diseases, WHO Collaborating Center for Osteoporosis Prevention, Geneva, University Hospital, 1211 Geneva, Switzerland \\ c Laboratory of Bone Imaging, Schulich School of Engineering, University of Calgary, Canada
}

\section{A R T I C L E I N F O}

\section{Article history:}

Received 10 May 2010

Accepted 27 August 2010

\section{Keywords:}

Bone adaptation

In-vivo mechanical loading

Bisphosphonate

Zoledronate

$\mu \mathrm{CT}$

\begin{abstract}
A B S T R A C T
Background: Local bisphosphonate delivery may be a solution to prevent periprosthetic bone loss and improve orthopedic implants fixation. In load-bearing implants, periprosthetic bone is exposed to high mechanical demands, which in normal conditions induce an adaptation of bone. In this specific mechanical situation, the modulation of the bone response by bisphosphonate remains uncertain.

Methods: We assessed the combined effects of zoledronate and mechanical loading on bone adaptation using an in-vivo axial compression model of the mouse tibia and injections of zoledronate. Bone structure was quantified with in-vivo $\mu \mathrm{CT}$ before and after the period of stimulation and the mechanical properties of the tibias were evaluated with 3 point-bending tests after sacrifice.

Findings: Axial loading induced a localized increase of cortical thickness and bone area. Zoledronate increased cortical thickness, bone perimeter, and bone area. At the most loaded site of the tibia, the combined effect of zoledronate and mechanical stimulation was significantly smaller than the sum of the individual effects measured at the same site in the control groups.

Interpretations: The results of this study suggested that a negative interaction between zoledronate and mechanical loading might exist at high level of strain.
\end{abstract}

(c) 2010 Elsevier Ltd. All rights reserved.

\section{Introduction}

Periprosthetic bone loss is initiated at the very early stage after an implant is set and facilitates the occurrence of aseptic loosening. Different authors have suggested the solution of local bisphosphonate release from the orthopedic implant to prevent the bone resorption at this early postoperative stage (Horowitz and Gonzales, 1996; Peter et al., 2001). It has been shown that local bisphosphonate preserves periprosthetic bone stock, in rats and sheep (Peter et al., 2005; Stadelmann et al., 2008; Wermelin et al., 2007) and also increases the fixation strength, in rats (Peter et al., 2005, 2006).

In these studies, the implants were not specifically loaded, in contrast to the clinical situation of load-bearing implants, where the periprosthetic bone is exposed to high mechanical demands (Huiskes et al., 1987). In this specific mechanical situation, the modulation of the bone response by bisphosphonate remains uncertain.

An early study showed that the effect of a dichloromethylene bisphosphonate on the bone apposition rate was increased when combined with a mechanical stimulation (Shellhart et al., 1992). On the other hand, by using 3-amino-1hydroxypropylidene-1-bisphosphonate,

\footnotetext{
* Corresponding author. EPFL/STI/IBI/LBO, Station 15, 1015 Lausanne, Switzerland. E-mail address: dominique.pioletti@epfl.ch (D.P. Pioletti).
}

Jagger et al. have shown that, in rats caudal vertebrae exposed to mechanical stimulus, the rate of bone apposition is not affected (Jagger et al., 1995). When fatigue loading is evaluated in rat bone, the use of alendronate did not protect the bone from fatigue in highly strained bone (Barrett et al., 2007). In another study, also in highly strained rat bone, alendronate was shown to suppress the apoptosis of osteocyte induced by the mechanical stimulation (Follet et al., 2007). The combination of mechanical loading and bisphosphonate, in particular zoledronate needs then to be further studied.

The aim of the present study was to assess the effect of zoledronate, the newer member of the third generation bisphosphonates (Green et al., 1996; Green, 1996; Pataki et al., 1997), on bone adaptation when a mechanical loading is applied. For this purpose, we used an in-vivo axial compression model of the mouse tibia (De Souza et al., 2005; Fritton et al., 2005, Stadelmann et al., 2009) and analyzed the effect of zoledronate on site-specific bone adaptation.

\section{Methods}

\subsection{Animals}

Eleven C57BL6 male mice, $17 \pm 1$ weeks old, were acclimated to our facility for three weeks. Mice were caged in groups of three or four. They were maintained under standard no barrier conditions and 
(a)

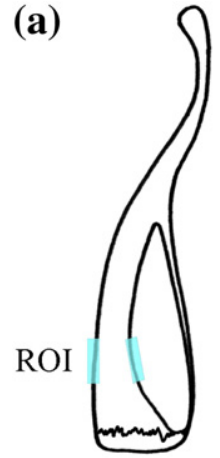

(b)

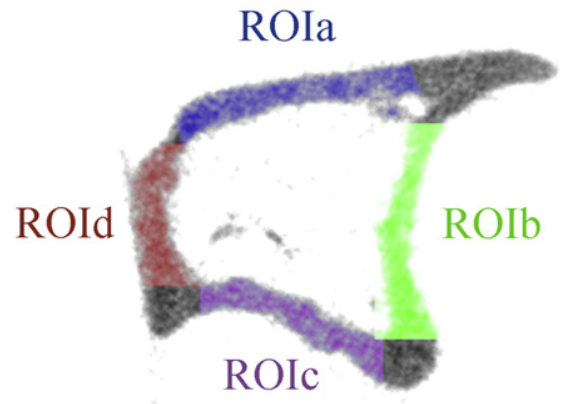

Fig. 1. Region of interest. Definition (a) of the global ROI and (b) of the 4 sub-ROI.

had access to mouse chow and water ad libidum. The local ethics committee on animal care approved all animal procedures (Protocol \#2006.1).

The mice were separated randomly into two groups: on day 0 , the five animals of the zoledronate group received a single subcutaneous injection $(80 \mu \mathrm{l})$ of $1 \mu \mathrm{g} / \mathrm{kg}$ zoledronate (Novartis Pharmaceuticals AG, Switzerland) while the six animals of the control group received an equivalent injection of saline.

\subsection{Anesthesia}

General anesthesia was induced with a ketamine $(80 \mathrm{mg} / \mathrm{kg})$ and xylazine $(5 \mathrm{mg} / \mathrm{kg})$ cocktail administered intraperitoneally unless specified.

\section{3. $\mu C T$}

We assessed bone architecture using in vivo micro-computed tomography ( $\mu$ CT1076 in vivo, SkyScan, Belgium) 11 days after zoledronate injection. Animals were anesthetized. The lower limbs were fixed in a custom polystyrene support and aligned with the axis of rotation of the scanner. The tibias were then scanned with $9 \mu \mathrm{m}$ isotropic voxel size, $50 \mathrm{kV}$ beam, $0.8^{\circ}$ step rotation. Reconstructions and analysis were performed with built-in routines of manufacturer's softwares NRecon and CTan, following the standard protocols. The reconstructed tibia contained about 1900 slices. Cortical thickness (Ct.Th), bone perimeter (B.Pm) and bone area (B.Ar) were evaluated at the proximal diaphysis ( $1 / 5$ of the tibial length) (Fig. 1a). This region of interest (ROI) was then divided in four sub-regions of interest (ROIa-d), corresponding to the four facets, to assess orientation specific remodeling (Fig. 1b).

\subsection{In vivo compression}

A compression machine was developed to apply controlled compression cycles on the tibias (Stadelmann et al., 2009), based on a previously published work (De Souza et al., 2005; Fritton et al., 2005). On day $1,3,5,8$ and 10 the left tibia of all animals were mechanically stimulated with dynamic axial compression sequences. Custom molded pads were designed on the axes end to apply the compression on the leg.

Each animal was anesthetized and placed on a warm support with eye gel until completely unresponsive. The animal was then placed on the stimulation machine with the left leg between the moving pad on the knee and the fixed pad on the ankle (Fig. 2a).

To maintain the initial position of the leg, a pre-load of $0.5 \mathrm{~N}$ was applied before the dynamic compression. The compression waveform was composed of square-like cycles at $2 \mathrm{~Hz}$ frequency, and amplitude from a force of $0.5 \mathrm{~N}$ during $0.25 \mathrm{~s}$ followed by a force of $8 \mathrm{~N}$ during $0.25 \mathrm{~s}$ (Fig. 2b). The sequence of compression was applied for $1 \mathrm{~min}$. Then the animal was placed at rest on the warm support. After $15 \mathrm{~min}$, a second sequence of $1 \mathrm{~min}$ of dynamic compressions was applied. The animal was again placed on the warm support until it moved.

Because of the natural curvature of the tibia, this simple axial loading induced combined compressive and bending strains. Axial compression of $8 \mathrm{~N}$ induces maximum octaedral shear strain at the postero-tibial crest $(1800 \mu \varepsilon \pm 40 \mu \varepsilon)$ and the antero-distal tibia (1940 $\mu \varepsilon \pm 30 \mu \varepsilon)$ (Stadelmann et al., 2009).

\subsection{Sacrifice and tibias extraction}

On day 11 , while still under anesthesia for the $\mu \mathrm{CT}$, the animals were sacrificed with an overdose of ketamin. Both tibias were extracted surgically and placed in wet conditions at $4{ }^{\circ} \mathrm{C}$.

\subsection{Mechanical tests}

Tibial mechanical properties were assessed by 3-point bending (Brodt et al., 1999; Jepsen et al., 2003), using the Instron Microtester 5848 (Instron, MA, USA), equipped with a $100 \mathrm{~N}$ gauge and custom bone supports. The fibula was removed with a surgical blade before the tests. The lower supports distance was set to $12 \mathrm{~mm}$ for all tibias, and the tibias always placed proximal end to the left and up, distal on the right. The crosshead speed was set to $0.02 \mathrm{~mm} / \mathrm{s}$ and the forcedisplacement data sampling to $100 \mathrm{~Hz}$.

The ultimate force, stiffness and post-yield energy to failure were calculated. The yield point was defined by using a $0.3 \mathrm{~N}$ offset from the stiffness line (Schriefer et al., 2005).

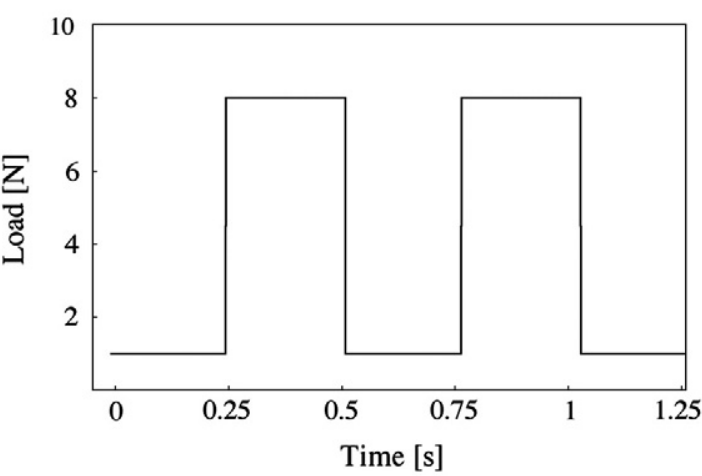

Fig. 2. (a) Animal's left tibia placed in the compression machine between the molded cups. (b) Compression waveform. 
Table 1

Number of tibias in each group.

\begin{tabular}{lll}
\hline & Force & \\
\cline { 2 - 3 } Zoledronate & $0 \mathrm{~N}$ (right) & $8 \mathrm{~N}$ (left) \\
\hline No $(1 \mu \mathrm{g} / \mathrm{kg}$ s.c.) & 6 & 6 \\
Yes & 5 & 5 \\
\hline
\end{tabular}

\subsection{Data analysis}

Data is presented as Mean \pm SD and all statistical procedures were performed with Mathematica5 ${ }^{\circledR}$ (Wolfram Research, USA). The number of tibias per group was accounted for as repetition of the measurement. Effects of mechanical loading and zoledronate were analyzed with two-way ANOVA and Tukey posthoc-tests. For independent data, Student $t$-test was used to determine the statistical power of the difference: $P<0.05$ was considered significant, while $P<0.1$ was considered as a strong trend.

\section{Results}

Twenty-two tibias were analyzed in this study (Table 1). Two tibias were damaged during extraction; they were excluded from mechanical analyses.

\subsection{Effect of mechanical loading and zoledronate on biomechanical parameters}

Mechanical loading alone significantly enhanced the tibial stiffness by $16 \%$ compared to control, but had no effect on ultimate force and post-yield energy to failure. Zoledronate significantly increased the stiffness, ultimate force and post-yield energy to failure by $31 \%, 24 \%$ and $60 \%$ respectively, compared to the control group. The effect of zoledronate on all three mechanical parameters was significantly higher than the effect of mechanical loading alone.

Mechanical loading combined with zoledronate significantly increased the biomechanical parameters, compared to control tibias. This effect was significantly higher than the effect of mechanical stimulation alone on ultimate force and post-yield energy to failure. However, no significant difference was observed between the effect of zoledronate and the combined effect of mechanical loading and zoledronate (Fig. 3).

Finally, for the three mechanical parameters, the sum of the effect of mechanical loading alone and the effect of zoledronate alone was not significantly different from the effect of mechanical loading with zoledronate. Note that no effect was detected on paired comparison of the mechanical parameters.

\subsection{Effect of mechanical loading and zoledronate on histomorphometric parameters}

For the histomorphometric analysis of the global ROI (Fig. 4 top), combination of zoledronate and mechanical loading had no statistical effect on B.Pm. A different behavior was observed for B.Ar as tibias with zoledronate and mechanical loading had significantly higher B.Ar compared to controls and mechanically stimulated ones. However these tibias were not significantly different that zoledronate-treated tibias. An effect was also statistically observed for Ct.Th in the global ROI between control and zoledronate-mechanical loading groups. When this parameter was further evaluated with respect to sub-ROI (Fig. 4 bottom), interestingly the value of $\mathrm{Ct}$.Th in ROIc of mechanically loaded tibias was significantly greater by $10 \%$ compared to controls, but no difference in other ROI was observed. Indeed in all sub-ROI except ROIc, the effect of combined zoledronate and mechanical stimulation was equivalent to the sum of the effect of zoledronate alone and the effect of mechanical stimulation alone. In ROIc, the effect of combined mechanical stimulation and zoledronate was significantly different from the sum of the effects.

\section{Discussion}

Local bisphosphonate release may be a solution to prevent periprosthetic bone loss and improve the implant fixation. However, in the case of load-bearing implants, periprosthetic bone is exposed to high mechanical demands, which in normal conditions induce an adaptation of bone. When zoledronate is present in bone, it alters its local metabolism. The aim of the study was to assess interactions between mechanical loading and zoledronate on bone adaptation in an in-vivo experiment.

We observed that axial mechanical loading induced a localized increase of cortical thickness and cortical bone area. These sitespecific changes are concordant with previous studies (De Souza et al., 2005; Fritton et al., 2005). Animals treated with zoledronate had sitespecific increased cortical thickness and cortical bone perimeter. The cortical bone area was increased in all zones, which is concordant with previous studies (Brouwers et al., 2008; Gasser et al., 2005; Recker et al., 2008).

In most cases, the effect of combined zoledronate and mechanical stimulation was equivalent to the sum of the effect of zoledronate and the effect of mechanical stimulation. However in ROIc, the combined effect of zoledronate and mechanical stimulation on structural properties was significantly smaller than the sum of the effects.

Using experimental measures and numerical simulations, we previously showed that during axial compression, bone in ROIc undergoes the highest strain levels (about $1900 \mu \varepsilon$ for $8 \mathrm{~N}$ load) while the rest of the tibia is less loaded (Stadelmann et al., 2009). The specific results in ROIc suggest that an interaction might exist between the bone response to mechanical stimulation and the effect of zoledronate at high levels of strain. The decreased effect of combined mechanical stimulation and zoledronate compared to mechanical stimulation alone, might reflect an upper limit on bone adaptation rate induced by the zoledronate when mechanical stimulations reach a high intensity.

This result seems to be contradicting previous studies that have assessed the impact of bisphosphonate on bone response to mechanical
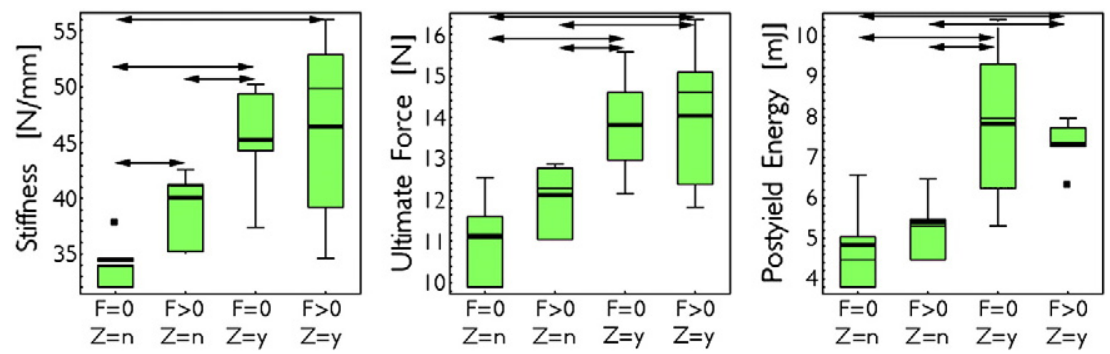

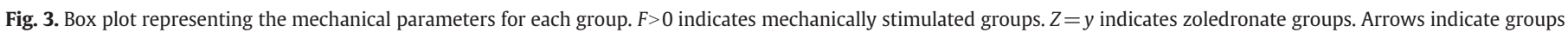
with significant differences $(P<0.05)$. Note the ordinates do not start at zero. 

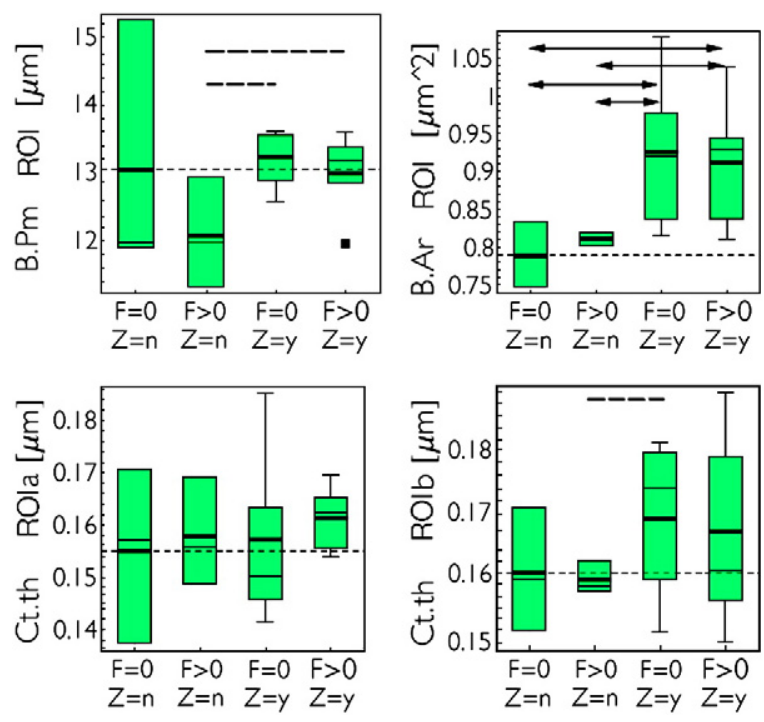
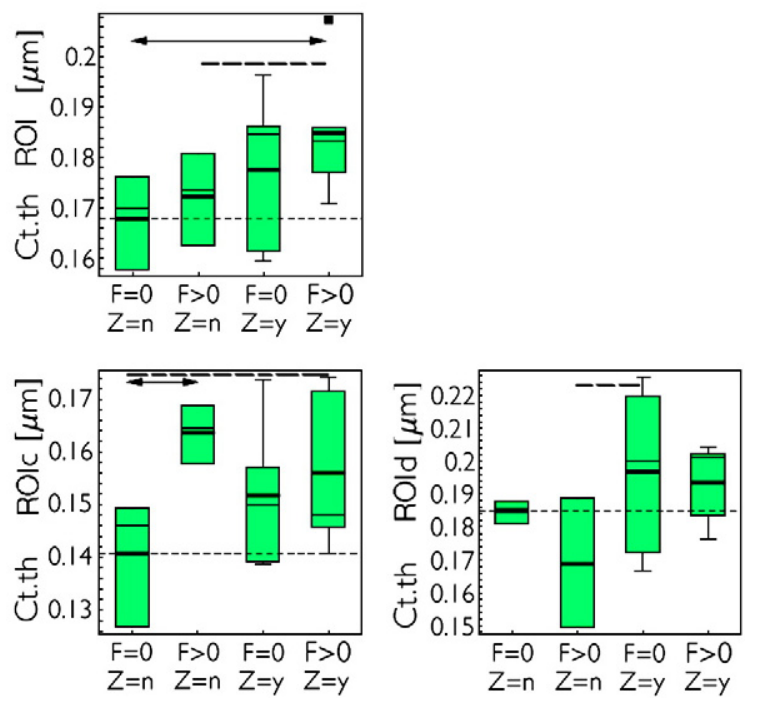

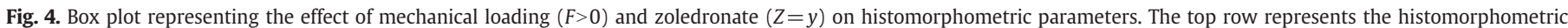

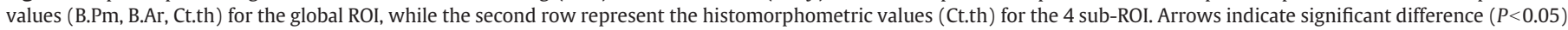
between groups, while dotted lines indicate strong trends of difference $(P<0.1)$. Note the ordinates do not start at zero.

demands. As examples, Jagger et al. have shown that, in rats' caudal vertebrae exposed to mechanical stimulus, the rate of bone apposition is not affected by bisphosphonate (Jagger et al., 1995). Braith et al. have shown that bone loss can be prevented by alendronate treatments and that alendronate combined with physiological mechanical loading induced a gain of bone mass (Braith et al., 2007). In the physiological range of strains (corresponding to strains in ROI a, b and d in our study), our data are consistent with Braith et al.'s results. At higher magnitudes of loading (corresponding to ROIc in our experiments), the use of bisphosphonate in combination with loading seems not as beneficial as loading alone. From the results presented in this study based on a limited number of rats, the use of bisphosphonate in situation of high strain level may affect the structure of the bone, or at least as found in previous studies using alendronate, may not protect the bone from fatigue (Barrett et al., 2007).

While the combined effect of mechanical loading and zoledronate has been suggested in a theoretical study (Pioletti and Rakotomanana, 2004), to our knowledge, no other data exist assessing the effect of zoledronate in situation involving high strain level. With interpretation valid only in rat so far, we could suggest that the possible limitation of the bone response to high mechanical stimulations by zoledronate would require a specific design of load-bearing implants, to prevent drug accumulation in high-loading areas of the periprosthetic bone.

\section{Acknowledgements}

Project no. 04-P2 was supported by the AO Research Fund of the AO Foundation, (Davos, Switzerland). We thank Novartis Pharma Research (Basel, Switzerland) for a generous gift of zoledronate. We thank Marc Jeanneret for his technical assistance. This work was supported by EC grant Health-2007, 2.4.5-3 (NB).

\section{References}

Barrett, J.G., Sample, S.J., McCarthy, J., Kalscheur, V.L., Muir, P., Prokuski, L., 2007. Effect of short-term treatment with alendronate on ulnar bone adaptation to cyclic fatigue loading in rats. J. Orthop. Res. 25, 1070-1077.

Braith, R.W., Conner, J.A., Fulton, M.N., Lisor, C.F., Casey, D.P., Howe, K.S., et al., 2007. Comparison of alendronate vs alendronate plus mechanical loading as prophylaxis for osteoporosis in lung transplant recipients: a pilot study. J. Heart Lung Transplant. 26, 132-137.
Brodt, M.D., Ellis, C.B., Silva, M.J., 1999. Growing C57B1/6 mice increase whole bone mechanical properties by increasing geometric and material properties. J. Bone Miner. Res. 14, 2159-2166.

Brouwers, J.E.M., Lambers, F.M., Gasser, J.A., Van Rietbergen, B., Huiskes, R., 2008. Bone degeneration and recovery after early and late bisphosphonate treatment of ovariectomized wistar rats assessed by in vivo micro-computed tomography. Calcif. Tissue Int. 82, 202-211.

De Souza, R.L., Matsuura, M., Eckstein, F., Rawlinson, S.C., Lanyon, L.E., Pitsillides, A.A., 2005. Non-invasive axial loading of mouse tibiae increases cortical bone formation and modifies trabecular organization: a new model to study cortical and cancellous compartments in a single loaded element. Bone 37, 810-818.

Follet, H., Li, J., Phipps, R.J., Hui, S., Condon, K., Burr, D.B., 2007. Risedronate and alendronate suppress osteocyte apoptosis following cyclic fatigue loading. Bone 40, 1172-1177.

Fritton, J.C., Myers, E.R., Wright, T.M., Van der Meulen, M.C.H., 2005. Loading induces site-specific increases in mineral content assessed by microcomputed tomography of the mouse tibia. Bone 36, 1030-1038.

Gasser, J.A., Ingold, P., Grosios, K., Laib, A., Hammerle, S., Kollr, B., 2005. Noninvasive monitoring of changes in structural cancellous bone parameters with a novel prototype micro-CT. J. Bone Miner. Metab. 23, 90-96.

Green, J., 1996. Zoledronate: the preclinical pharmacology. Br. J. Clin. Pract. 16-18.

Green, J.R., Hornby, S.B., Evans, G.P., Muller, K., 1996. Effect of 1-year treatment with zoledronate (CGP 42446) on bone mineral density, bone mechanical properties and biochemical markers in the ovariectomized rat. J. Bone Miner. Res. 11, M631.

Horowitz, S.M., Gonzales, J.B., 1996. Inflammatory response to implant particulates in a macrophage/osteoblast coculture model. Calcif. Tissue Int. 59, 392-396.

Huiskes, R., Weinans, H., Grootenboer, H.J., Dalstra, M., Fudala, B., Slooff, T.J., 1987. Adaptive bone-remodeling theory applied to prosthetic-design analysis. J. Biomech. 20, 1135-1150.

Jagger, C.J., Chambers, T.J., Chow, J.W.M., 1995. Stimulation of bone-formation by dynamic-mechanical loading of rat caudal vertebrae is not suppressed by 3-Amino1-Hydroxypropylidene-1-Bisphosphonate (Ahprbp). Bone 16, 309-313.

Jepsen, K.J., Akkus, O., Majeska, R.J., Nadeau, J.H., 2003. Hierarchical relationship between bone traits and mechanical properties in inbred mice. Mamm. Genome 14, 97-104.

Pataki, A., Muller, K., Green, J.R., Ma, Y.F., Li, Q.N., Jee, W.S.S., 1997. Effects of short-term treatment with the bisphosphonates zoledronate and pamidronate on rat bone: a comparative histomorphometric study on the cancellous bone formed before, during, and after treatment. Anat. Rec. 249, 458-468.

Peter, B., Pioletti, D.P., Terrier, A., Rakotomanana, L.R., 2001. Orthopaedic implant as drug delivery system: a numerical approach. Comput. Meth. Biomech. Biomed. Eng. 4, 505-513.

Peter, B., Pioletti, D.P., Laib, S., Bujoli, B., Pilet, P., Janvier, P., et al., 2005. Calcium phosphate drug delivery system: influence of local zoledronate release on bone implant osteointegration. Bone 36, 52-60.

Peter, B., Gauthier, O., Laib, S., Bujoli, B., Guicheux, J., Janvier, P., et al., 2006. Local delivery of bisphosphonate from coated orthopedic implants increases implants mechanical stability in osteoporotic rats. J. Biomed. Mater. Res. A 76, 133-143.

Pioletti, D.P., Rakotomanana, L.R., 2004. Can the increase of bone mineral density following bisphosphonates treatments be explained by biomechanical considerations? Clin Biomech (Bristol, Avon) 19, 170-174.

Recker, R.R., Delmas, P.D., Halse, J., Reid, I.R., Boonen, S., Garcia-Hernandez, P.A., et al., 2008. Effects of intravenous zoledronic acid once yearly on bone remodeling and bone structure. J. Bone Miner. Res. 23, 6-16. 
Schriefer, J.L., Robling, A.G., Warden, S.J., Fournier, A.J., Mason, J.J., Turner, C.H., 2005. A comparison of mechanical properties derived from multiple skeletal sites in mice. J. Biomech. 38, 467-475.

Shellhart, W.C., Hardt, A.B., Moore, R.N., Erickson, L.C., 1992. Effects of bisphosphate treatment and mechanical loading on bone modeling in the rat tibia. Clin Orthop Relat Res 253-259.

Stadelmann, V.A., Gauthier, O., Terrier, A., Bouler, J.M., Pioletti, D.P., 2008. Implants delivering bisphosphonate locally increase periprosthetic bone density in an osteoporotic sheep model. A pilot study. Eur. Cell Mater. 16, 10-16.
Stadelmann, V.A., Hocke, J., Verhelle, J., Forster, V., Merlini, F., Terrier, A., et al., 2009. 3D strain map of axially loaded mouse tibia: a numerical analysis validated by experimental measurements. Comput Methods Biomech Biomed Eng 12, 95-100.

Wermelin, K., Tengvall, P., Aspenberg, P., 2007. Surface-bound bisphosphonates enhance screw fixation in rats - increasing effect up to 8 weeks after insertion. Acta Orthop. 78, 385-392. 